PROCEEDINGS OF THE AMERICAN MATHEMATICAL SOCIETY

Volume 135, Number 3, March 2007, Pages 755-766

S 0002-9939(06)08524-8

Article electronically published on August 31, 2006

\title{
EXISTENCE AND NONEXISTENCE OF HYPERCYCLIC SEMIGROUPS
}

\author{
L. BERNAL-GONZÁLEZ AND K.-G. GROSSE-ERDMANN
}

(Communicated by Joseph A. Ball)

\begin{abstract}
In these notes we provide a new proof of the existence of a hypercyclic uniformly continuous semigroup of operators on any separable infinitedimensional Banach space that is very different from - and considerably shorter than - the one recently given by Bermúdez, Bonilla and Martinón. We also show the existence of a strongly dense family of topologically mixing operators on every separable infinite-dimensional Fréchet space. This complements recent results due to Bès and Chan. Moreover, we discuss the Hypercyclicity Criterion for semigroups and we give an example of a separable infinitedimensional locally convex space which supports no supercyclic strongly continuous semigroup of operators.
\end{abstract}

\section{INTRODUCTION}

Assume that $X$ is a topological vector space over the field $\mathbb{K}=\mathbb{R}$ or $\mathbb{C}$. Let $L(X)$ denote the space of all operators on $X$, that is, all continuous linear mappings $X \rightarrow X$. Then an operator $T \in L(X)$ is called hypercyclic whenever there exists some $x \in X$ such that the orbit $\left\{T^{n} x: n \in \mathbb{N}\right\}$ of $x$ under $T$ is dense in $X$. In this case the vector $x$ is also called hypercyclic. The theory of hypercyclic operators has recently been studied intensively. We refer to the comprehensive survey [28] (see also [24, Section 1], 30, [13] and [37]). More generally, a sequence $\left(T_{n}\right)_{n \geq 1}$ of operators on $X$ is called hypercyclic provided there exists some $x \in X$ such that $\left\{T_{n} x: n \in \mathbb{N}\right\}$ is dense in $X$. Observe that $X$ must be separable in order to support such a sequence. We continue to refer to the set $\left\{T_{n} x: n \in \mathbb{N}\right\}$ as the orbit of $x$ under $\left(T_{n}\right)$. A vector $x$ with dense orbit under $\left(T_{n}\right)$ is called hypercyclic for $\left(T_{n}\right)$. This more general notion of hypercyclicity is also sometimes referred to as universality (see [28, Section 1]).

The Hypercyclicity Criterion, which gives sufficient conditions under which a sequence $\left(T_{n}\right)$ is hypercyclic, has turned out to be extremely useful in applications (see, for instance, [6], 24], 29] and [36]). Recall that an F-space is a topological vector space whose topology is induced by a complete translation-invariant metric. A Fréchet space is a locally convex F-space.

Received by the editors November 29, 2004 and, in revised form, October 5, 2005.

2000 Mathematics Subject Classification. Primary 47A16; Secondary 47D03.

Key words and phrases. Hypercyclic uniformly continuous semigroup of operators, topologically mixing semigroup, Hypercyclicity Criterion, supercyclic semigroup.

The first author was partially supported by Plan Andaluz de Investigación de la Junta de Andalucía FQM-127 and by Ministerio de Ciencia y Tecnología Grant BFM2003-03893-C02-01.

(C)2006 American Mathematical Society Reverts to public domain 28 years from publication 
Definition 1.1. Let $X$ be a separable F-space. A sequence $\left(T_{n}\right) \subset L(X)$ satisfies the Hypercyclicity Criterion provided there exist dense subsets $X_{0}$ and $Y_{0}$ of $X$ and an increasing sequence $\left(n_{k}\right)$ of positive integers satisfying the following two conditions:

(i) $T_{n_{k}} x \rightarrow 0(k \rightarrow \infty)$ for all $x \in X_{0}$;

(ii) for any $y \in Y_{0}$ there is a sequence $\left(u_{k}\right)$ in $X$ such that $u_{k} \rightarrow 0$ and $T_{n_{k}} u_{k} \rightarrow$ $y(k \rightarrow \infty)$.

The proof that $\left(T_{n}\right)$ is hypercyclic under these conditions is an application of the Baire category theorem (see, for example, [28, Theorem 2]). A corresponding concept can be defined for a single operator $T \in L(X)$ by looking at its sequence $\left(T^{n}\right)$ of iterates. Diverse versions of the criterion are stated in [33, 23, Remark 2.3], 27, [24, Corollary 1.4] and [10, Definition 1.2 and Remark 2.6]; we refer to 41] and [4] for a comparison of these versions. For properties that are equivalent to the Hypercyclicity Criterion see [10, 7], 34, 42] and [26.

In recent years there has been considerable interest in the hypercyclicity of semigroups of operators. A strongly continuous semigroup (see, for instance, 20, Chapter VIII] or [22]) on a topological vector space $X$ is a family $(T(t))_{t \geq 0} \subset L(X)$ such that

(i) $T(0)=I$ and $T(t+s)=T(t) T(s)$ for $t, s \geq 0$;

(ii) the map $t \in[0, \infty) \mapsto T(t) x \in X$ is continuous for every $x \in X$. In other words, the map $t \mapsto T(t) \in L(X)$ is continuous when $L(X)$ is endowed with the strong operator topology (SOT).

If, in addition, $X$ is a normed space and the map $t \in[0, \infty) \mapsto T(t) \in L(X)$ is continuous in the uniform operator topology, then the family $(T(t))_{t \geq 0}$ is called a uniformly continuous semigroup. According to Desch, Schappacher and Webb [19], a semigroup $(T(t))_{t \geq 0}$ is called hypercyclic if there exists a hypercyclic vector, that is, some $x \in X$ such that the orbit $\{T(t) x: t \geq 0\}$ is dense in $X$; and it is called chaotic if, in addition, there is a dense set of periodic points, that is, points $x \in X$ such that $T(t) x=x$ for some $t>0$.

Oxtoby and Ulam [40, Theorem 6 ] have shown that for any hypercyclic strongly continuous semigroup $(T(t))_{t \geq 0}$ on an F-space $X$ there is some $t>0$ such that the operator $T(t)$ is hypercyclic, and the set of these $t$ is residual in $(0, \infty)$. Conejero [16] has recently improved the result by showing that, in fact, if $x \in X$ is a hypercyclic vector for $(T(t))_{t \geq 0}$, then there is a residual set $A \subset(0, \infty)$ such that $x$ is hypercyclic for each operator $T(t)$ with $t \in A$. In this context we recall the following; cf. 3, Open Problem 1].

Problem 1.2. If $(T(t))_{t>0}$ is a hypercyclic strongly continuous semigroup on an F-space $X$, are then all $T(t), t>0$, hypercyclic operators?

In these notes, we provide a proof of the existence of a hypercyclic uniformly continuous semigroup of operators on any separable infinite-dimensional Banach space (see Section 3). This proof is very different from the one recently given by Bermúdez, Bonilla and Martinón [3]. The more restrictive class of topologically mixing semigroups will be considered in Section 4. An example of a separable infinite-dimensional locally convex space that supports no supercyclic (hence no hypercyclic) strongly continuous semigroup is furnished in Section 5. There we also show that the analogue of Problem 1.2 for real supercyclic semigroups has a 
negative solution. We begin, in Section 2, with a discussion of the Hypercyclicity Criterion for semigroups of operators.

\section{The Hypercyclicity CRITERION FOR SEMigroups of OPERATORS}

There is a natural analogue of Definition 1.1 for semigroups that was recently introduced by El Mourchid 21] and Conejero and Peris 17] (see also [19, Theorem $2.3])$.

Definition 2.1. Let $X$ be a separable F-space. A strongly continuous semigroup $(T(t))_{t \geq 0}$ on $X$ satisfies the Hypercyclicity Criterion provided there exist dense subsets $X_{0}$ and $Y_{0}$ of $X$ and an increasing sequence $\left(t_{n}\right) \subset[0, \infty)$ satisfying the following two conditions:

(i) $T\left(t_{n}\right) x \rightarrow 0(n \rightarrow \infty)$ for all $x \in X_{0}$;

(ii) for any $y \in Y_{0}$ there is a sequence $\left(u_{n}\right)$ in $X$ such that $u_{n} \rightarrow 0$ and $T\left(t_{n}\right) u_{n} \rightarrow y(n \rightarrow \infty)$.

As in the discrete case, one can show that a strongly continuous semigroup $(T(t))_{t \geq 0}$ is hypercyclic whenever it satisfies the Hypercyclicity Criterion (see [21]). However, this is also a consequence of the corresponding result for sequences $\left(T_{n}\right)$ of operators and the following simple observation (cf. also Conejero [16, Chapter $6])$.

Observation 2.2. Let $(T(t))_{t \geq 0}$ be a strongly continuous semigroup of operators on a separable $F$-space $X$. Then we have the following:

(a) $(T(t))_{t>0}$ is hypercyclic if and only if $\left(T\left(t_{n}\right)\right)$ is a hypercyclic sequence for some increasing sequence $\left(t_{n}\right) \subset[0, \infty)$.

(b) $(T(t))_{t \geq 0}$ satisfies the Hypercyclicity Criterion if and only if the sequence $\left(T\left(t_{n}\right)\right)$ satisfies the Hypercyclicity Criterion for some increasing sequence $\left(t_{n}\right) \subset$ $[0, \infty)$.

In this way certain results on hypercyclic sequences $\left(T_{n}\right)$ translate immediately to semigroups. In particular we obtain the following characterization of the Hypercyclicity Criterion as an application of Theorems 3.3 and 3.4 of our earlier investigation [7]. We recall that a sequence $\left(T_{n}\right)$ is called hereditarily hypercyclic if every subsequence $\left(T_{n_{k}}\right)$ is hypercyclic.

Theorem 2.3. Let $(T(t))_{t \geq 0}$ be a strongly continuous semigroup of operators on a separable $F$-space $X$. Then the following assertions are equivalent:

(i) $(T(t))_{t \geq 0}$ satisfies the Hypercyclicity Criterion.

(ii) $(T(t))_{t \geq 0}$ has a hereditarily hypercyclic subsequence $\left(T\left(t_{n}\right)\right)$.

(iii) $(T(t) \oplus T(t))_{t>0}$ is hypercyclic on $X \times X$.

(iv) For every pair $U, V$ of nonempty open subsets of $X$ and every neighbourhood $W$ of zero there is some $t \geq 0$ with $T(t)(U) \cap W \neq \emptyset$ and $T(t)(W) \cap V \neq \emptyset$.

The equivalence of (i) and (iii) was also obtained, independently and with a different proof, by Conejero and Peris [17.

Grivaux [26] has shown that a hypercyclic operator on a Banach space satisfies the Hypercyclicity Criterion if it has a dense set of points whose orbits are bounded. By the Oxtoby-Ulam theorem mentioned in the Introduction, the result carries over to semigroups. We will give here a direct proof based on the idea of proof of [26]. 
Theorem 2.4. Let $(T(t))_{t \geq 0}$ be a hypercyclic strongly continuous semigroup of operators on an F-space $X$. If there is a dense set of points $x \in X$ with bounded orbits $\{T(t) x: t \geq 0\}$, then $(T(t))_{t \geq 0}$ satisfies the Hypercyclicity Criterion.

Proof. We claim that condition (iv) of Theorem 2.3 is satisfied. Let $U, V$ be nonempty open subsets of $X$ and $W$ a neighbourhood of zero. By assumption, there is a point $x_{0} \in U$ whose orbit is bounded, hence there is some $\rho>0$ such that $\rho T(t) x_{0} \in W$ for all $t \geq 0$. On the other hand, by hypercyclicity of $(T(t))$ we have that $\rho T\left(t_{0}\right) W \cap V \neq 0$ for some $t_{0} \geq 0$. Then, by continuity of $T\left(t_{0}\right)$ and the density of hypercyclic vectors there is a hypercyclic vector $x \in U$ such that $\rho T\left(t_{0}\right) x \in W$. By hypercyclicity of $x$ there is some $s>0$ such that $T(s) x \in W$ and $\rho T\left(t_{0}\right) T(s) x \in V$. In addition we can approximate $\rho T\left(t_{0}\right) x$ arbitrarily closely by suitable $T(r) x$. Hence there is some $r>0$ such that $T(r) x \in W$ and $T(s) T(r) x \in V$. This shows that $T(r) U \cap W \neq 0$ and $T(r) W \cap V \neq 0$, which proves the claim.

In particular, every chaotic strongly continuous semigroup of operators on an F-space $X$ satisfies the Hypercyclicity Criterion.

As we mentioned in the Introduction it is an open problem if for a hypercyclic strongly continuous semigroup $(T(t))_{t \geq 0}$ every operator $T(t), t>0$, hence every sequence $(T(n t))$, is hypercyclic. If, instead, we ask for the hypercyclicity of any subsequence $\left(T\left(t_{n}\right)\right)$ with $t_{n} \rightarrow \infty$ and $\sup _{n>1}\left(t_{n+1}-t_{n}\right)<\infty$, then the property turns out to be equivalent to the Hypercyclicity Criterion. This follows from the recent solution of the Bounded Steps Problem (see [42] and [26]).

Theorem 2.5. Let $(T(t))_{t \geq 0}$ be a strongly continuous semigroup of operators on a separable $F$-space $X$. Then $\left(T\left(t_{n}\right)\right)$ is hypercyclic for any increasing sequence $\left(t_{n}\right)$ with $t_{n} \rightarrow \infty$ and $\sup _{n \geq 1}\left(t_{n+1}-t_{n}\right)<\infty$ if and only if $(T(t))_{t \geq 0}$ satisfies the Hypercyclicity Criterion.

Proof. We assume that $(T(t))_{t \geq 0}$ satisfies the Hypercyclicity Criterion with respect to the sequence $\left(\tau_{n}\right) \subset[0, \infty)$. Let $\left(t_{n}\right)$ be an increasing sequence with $t_{n} \rightarrow \infty$ and $\sup _{n \geq 1}\left(t_{n+1}-t_{n}\right)<\infty$. Then there are $M>0, m_{n} \in \mathbb{N}$ and $\delta_{n} \geq 0$ with $\delta_{n} \leq M$ such that $\tau_{n}=t_{m_{n}}-\delta_{n}, n \geq 1$. Hence we have that $T\left(t_{m_{n}}\right) x=T\left(\delta_{n}\right) T\left(\tau_{n}\right) x$ for all $x \in X$. Without loss of generality we may assume that $\delta_{n}$ converges to some $\delta \in[0, M]$. This easily implies that the sequence $\left(T\left(t_{n}\right)\right)$ satisfies the Hypercyclicity Criterion and hence is hypercyclic. We need only observe that, by pointwise continuity, the family $(T(t))_{0 \leq t \leq M}$ is equicontinuous so that $T\left(\delta_{n}\right) x_{n} \rightarrow T(\delta) x$ if $x_{n} \rightarrow x$, and, by hypercyclicity of $(T(t))_{t \geq 0}, T(\delta)$ has dense range so that $T(\delta)(Y)$ is dense in $X$ whenever $Y \subset X$ is dense.

Conversely, the hypothesis implies in particular that $\left(T\left(m_{n}\right)\right)$ is hypercyclic for any strictly increasing sequence $\left(m_{n}\right)$ of positive integers with bounded differences. The solution of the Bounded Steps Problem [42, Theorem 2.3] shows that the operator $T(1)$ satisfies the Hypercyclicity Criterion, hence so does $(T(t))_{t \geq 0}$.

Remark 2.6. In this context we might note that, trivially, there is no version of Theorem 2.5 for sequences of operators, even if they are commuting. To see this, let $S$ be an operator that satisfies the Hypercyclicity Criterion and define, for $n \geq 1, T_{2 n-1}=S^{n}$ and $T_{2 n}=I$, the identity operator. Then $\left(T_{n}\right)$ satisfies the Hypercyclicity Criterion but $\left(T_{2 n}\right)$ is not hypercyclic. 


\section{EXISTENCE OF HYPERCYCLIC SEMIGROUPS}

In the setting of separable infinite-dimensional Banach spaces, Rolewicz [4] had posed the problem of whether every such space supports a hypercyclic operator $T$. The problem was solved in the affirmative by Ansari [1] and the first author [5] (see also [14). The operator $T$ can be constructed to be a compact perturbation of the identity $I$, that is, $T=I+K$, where $K$ is a compact operator. In addition, $T$ can be selected so that it satisfies the Hypercyclicity Criterion; cf. [35. p. 530].

Bermúdez, Bonilla and Martinón [3, Theorem 2.4] have recently established the existence of a hypercyclic uniformly continuous semigroup of operators on any separable infinite-dimensional Banach space in the case $\mathbb{K}=\mathbb{C}$. Their proof, which is based on results due to Desch, Schappacher and Webb [19, Martínez-Giménez and Peris [36], and Ovsepian and Pełczyński [39], seems to work also for real Banach spaces. We shall obtain here the same result with a proof that is quite different from (and considerably shorter than) that of [3].

Theorem 3.1. Every separable infinite-dimensional Banach space $X$ supports a uniformly continuous semigroup $(T(t))_{t \geq 0}$ satisfying the Hypercyclicity Criterion. In particular, $(T(t))_{t \geq 0}$ is hypercyclic.

Proof. We first select an operator $S=I+K \in L(X)$ satisfying the Hypercyclicity Criterion such that $K$ is compact. At this point we distinguish the cases $\mathbb{K}=\mathbb{C}$ and $\mathbb{K}=\mathbb{R}$.

Assume that $\mathbb{K}=\mathbb{C}$. Then by a result due to Chan and Shapiro [15, p. 1446] we have that the spectrum of $K$ is $\sigma(K)=\{0\}$, so $\sigma(S)=\{1\}$. Therefore the origin belongs to the unbounded connected component of the complement of $\sigma(S)$, hence $S$ has a logarithm in the Banach algebra $L(X)$ (see [44, Theorem 10.30]), that is, there is an operator $A \in L(X)$ such that

$$
\exp A:=\sum_{n=0}^{\infty} \frac{A^{n}}{n !}=S
$$

Let us define $T(t):=\exp (t A)(t \geq 0)$, which is clearly a uniformly continuous semigroup of operators on $X$. But if we take $t_{n}=n(n \in \mathbb{N})$, then the sequence $\left(T\left(t_{n}\right)\right)=\left(S^{n}\right)$ satisfies the Hypercyclicity Criterion, hence so does $(T(t))_{t \geq 0}$ by Observation 2.2 .

As for the case $\mathbb{K}=\mathbb{R}$, let us consider the complexification $\widetilde{X}=X+i X$ of $X$ and the complexification $\widetilde{S}$ of $S$, that is, $\widetilde{S}(x+i y)=S x+i S y$. Then $\widetilde{X}$ is a complex Banach space if we endow it with, for instance, the Taylor norm $\|x+i y\|_{\tau}=\sup _{0 \leq t \leq 2 \pi}\|x \cos t-y \sin t\|$, and for $R \in L(X)$ we have that the operator norms of $R$ and of its complexification $\widetilde{R}$ satisfy $\|\widetilde{R}\|=\|R\|$ (see, for instance, [38]). From Corollary 2.8 of [10] we have that $\widetilde{S}$ satisfies the Hypercyclicity Criterion, so it is hypercyclic. Since the constructions in [1] and [5] work equally when we replace $K$ by any $\lambda K, \lambda \neq 0$, we may assume that $\|K\|=\|\widetilde{K}\|<1$. At this point we combine Dunford's symbolic calculus (see, for instance, 44]) with complexification. Consider the operator

$$
A:=\sum_{n=1}^{\infty} \frac{(-1)^{n+1}}{n} K^{n} \in L(X)
$$


The operator $\sum_{n=1}^{\infty} \frac{(-1)^{n+1}}{n}(\widetilde{K})^{n}$ is a well-defined member of $L(\widetilde{X})$ and equals $\widetilde{A}$. But the principal branch $\varphi$ of the logarithm of $1+z$ satisfies $\varphi(z)=\sum_{n=1}^{\infty} \frac{(-1)^{n+1}}{n} z^{n}$ on the open unit disk of $\mathbb{C}$ and, trivially, $\exp (\varphi(z))=1+z$. Therefore $\exp (\widetilde{A})=$ $\widetilde{I}+\widetilde{K}=\widetilde{S}$, that is, $\sum_{n=0}^{\infty} \frac{(\widetilde{A})^{n}}{n !}=\widetilde{S}$. If we apply this equality to the vectors of the form $x+i 0$ of $\widetilde{X}$ and take into account that $(\widetilde{A})^{n}=\widetilde{A^{n}}$ for all $n$, then we get that $\exp A=\sum_{n=0}^{\infty} \frac{A^{n}}{n !}=S$. When we now define $T(t):=\exp (t A)(t \geq 0)$ the conclusion follows as in the complex case.

Remarks 3.2. (a) The operator $A$ such that $T(t)=\exp (t A)$ obtained in the preceding proof is the infinitesimal generator of $(T(t))_{t \geq 0}$. In fact, a semigroup of operators on a Banach space $X$ is uniformly continuous if and only if it has the form $\exp (t A)$ for some $A \in L(X)$; cf. [20, Chapter VIII] or [22. Of course, in the first part of the proof we could have defined the operator $A$ explicitly, exactly as in the second part.

(b) In [3, Theorem 3.3] it is proved that there are separable infinite-dimensional Banach spaces that admit no chaotic strongly continuous semigroups. This is analogous to a negative result on chaotic operators due to Bonet, Martínez-Giménez and Peris [12].

(c) Bonet and Peris 14 were able to show that every separable infinite-dimensional Fréchet space supports a hypercyclic operator. Conejero [16] has recently proved that each such space, different from the space $\omega=\mathbb{K}^{\mathbb{N}}$ of all scalar sequences, also admits a hypercyclic (and even mixing, see Section 4) strongly continuous semigroup, while in the special case of $\mathbb{K}^{\mathbb{N}}$ there is no hypercyclic semigroup of the form $T(t)=\exp (t A), t \geq 0$, for some $A \in L(\omega)$.

Solving a problem of Halperin, Kitai and Rosenthal, Grivaux [25] has shown that every linearly independent sequence of vectors in a separable infinite-dimensional Banach space is contained in the orbit of the first vector under some operator. Since Grivaux constructs such an operator in the form $I+K$, where $K$ is compact, the proof of Theorem 3.1 immediately gives the following.

Theorem 3.3. Let $\left(x_{n}\right)_{n \geq 0}$ be a linearly independent sequence of vectors in a separable infinite-dimensional Banach space $X$. Then there exists a uniformly continuous semigroup $(T(t))_{t \geq 0}$ on $X$ such that each $x_{n}, n \geq 1$, is contained in the orbit of $x_{0}$ under $(T(t))_{t \geq 0}$.

\section{Topologically miXing operators on FrÉCHET SPACES}

During the preparation of an earlier version of this paper we were kindly informed by A. Bonilla that in a recent joint work with Bermúdez, Conejero and Peris [2, Theorem 2.4] it was shown that on every separable infinite-dimensional Banach space there exists a uniformly continuous semigroup of operators $(T(t))_{t \geq 0}$ that is not only hypercyclic but also topologically mixing. (In fact, such a semigroup can even be chosen to be topologically mixing in the right half-plane; see [2] for details.)

An operator $T \in L(X)$ on a separable $F$-space $X$ is said to be topologically mixing if for any pair of nonempty open subsets $U, V$ of $X$ there exists $N \in \mathbb{N}$ such that $T^{n}(U) \cap V \neq \emptyset$ for all $n \geq N$. It is well known that $T$ is hypercyclic if and only if it is topologically transitive in the sense of Birkhoff, that is, if for any pair of nonempty open subsets $U, V$ of $X$ there exists $N \in \mathbb{N}$ such that $T^{N}(U) \cap V \neq \emptyset$ 
(see [28]). Thus every topologically mixing operator is hypercyclic. A semigroup $(T(t))_{t \geq 0}$ of operators on $X$ is said to be topologically mixing when for any pair of nonempty open subsets $U, V$ of $X$ there exists $t_{0}>0$ such that $T(t)(U) \cap V \neq \emptyset$ for all $t \geq t_{0}$. It is easy to see that if $(T(t))_{t \geq 0}$ is topologically mixing, then every single operator $T(t)(t>0)$ is topologically mixing, hence hypercyclic, from which it is trivial that $(T(t))_{t \geq 0}$ is hypercyclic as a semigroup.

Hence the mentioned result of 2 improves [3, Theorem 2.4]. One also derives that every separable infinite-dimensional Banach space supports a topologically mixing operator. This has also been obtained, independently, by S. Grivaux [26, Theorem 2.6]. We point out that there are Banach spaces (for instance, the space $l_{p}$ of scalar sequences with absolutely summable $p$-power, where $1 \leq p<\infty$ ) that support hypercyclic operators which are not topologically mixing; see [18] and [26].

We will show here that every separable infinite-dimensional Fréchet space $X$ admits a topologically mixing operator. In fact, using recent ideas of Bès and Chan, we even obtain that the set of such operators is SOT-dense in $L(X)$.

Bès and Chan had proved [8, Theorem 2] that the set of hypercyclic operators on $L(X)$ is not only nonempty (see [14]) but even SOT-dense. Then they realized 9 , Corollary 6$]$ that the proof could be considerably simplified by using the following result that is essentially due to Hadwin, Nordgren, Radjavi and Rosenthal (31; see also [9, Theorem 1 and the note following it]).

Lemma 4.1. Let $X$ be an infinite-dimensional locally convex space, and let $T \in$ $L(X)$. Then the following statements are equivalent:

(i) The set of conjugates $\left\{S T S^{-1}: S\right.$ invertible $\}$ of $T$ is SOT-dense in $L(X)$.

(ii) For all $n \in \mathbb{N}$, there exist vectors $x_{1}, \ldots, x_{n}$ in $X$ so that the set $\left\{x_{1}, \ldots, x_{n}, T x_{1}, \ldots, T x_{n}\right\}$ is linearly independent.

We will employ the lemma to prove the main result of this section. As can be observed, its proof is independent of the mentioned result of Bermúdez et al. 2, Theorem 2.4].

Theorem 4.2. Let $X$ be a separable infinite-dimensional Fréchet space. Then the class $\mathcal{M}$ of surjective topologically mixing operators on $X$ is SOT-dense in $L(X)$, in particular nonempty.

Proof. It is clear that the class $\mathcal{M}$ is invariant under conjugation. On the other hand, if $T$ is topologically mixing, then it is hypercyclic, so condition (ii) in Lemma 4.1 is fulfilled; indeed, pick a hypercyclic vector $x$ for $T$ and choose $x_{1}=x, x_{2}=$ $T^{2} x, \ldots, x_{n}=T^{2 n-2} x$ for each $n \in \mathbb{N}$. Thus, it is enough to exhibit a surjective topologically mixing operator $T$ on $X$.

In fact, such operators have already been constructed by Bonet and Peris 14, Theorem 1]. If $X=\omega$, then the backward shift $B$, given by $B\left(x_{k}\right)=\left(x_{k+1}\right)$, is surjective, and it is topologically mixing: by the first part of the proof of [14, Theorem 1] it satisfies Criterion 3.1 of [2], which also holds for F-spaces.

For $X \neq \omega$, the proof of Theorem 1 in [14] constructs a surjective hypercyclic operator $T$ on $X$. In addition, the operator $\widetilde{T}: l_{1} \rightarrow l_{1}$ defined there is topologically mixing by [26, Lemma 2.3], hence so is $T$ as follows from [2, Lemma 2.2], which also holds for Fréchet spaces.

By [2, Theorem 3.5], which also holds for Fréchet spaces, a strongly continuous semigroup $(T(t))_{t \geq 0}$ is topologically mixing if and only if some $T(t), t>0$, 
is topologically mixing. Hence, the existence of topologically mixing operators on separable infinite-dimensional Fréchet spaces other than $\mathbb{K}^{\mathbb{N}}$ also follows from Conejero's result mentioned in Remark 3.2(c). Conversely, we note the following.

Remark 4.3. In the case of separable infinite-dimensional Banach spaces the construction in the proof of Theorem 4.2 can be used to obtain a new proof of the existence of topologically mixing uniformly continuous semigroups. It suffices to use the procedure of the proof of Theorem 3.1 and to apply [2, Theorem 3.5].

\section{NoneXistenCE OF HYPERCYCLIC SEMIGROUPS}

It is well known that no operator on a finite-dimensional topological vector space $X$ is hypercyclic (see [43, p. 17]). But even on a separable infinite-dimensional topological vector space the family of hypercyclic operators may be empty. This can happen, for instance, in the class of complete LB-spaces, that is, complete inductive limits of Banach spaces. In fact, the second author [28, Remark 4(a)] showed that the countable direct sum $\varphi=\bigoplus_{n \in \mathbb{N}} \mathbb{K}$ of copies of the scalar field equipped with the natural inductive topology does not support any hypercyclic operators. The same result was also obtained by Bonet and Peris [14. Proposition 6], who even show that no operator on $\varphi$ can be supercyclic. Recall that an operator $T \in L(X)$ is supercyclic if there is a vector $x \in X$-called supercyclic for $T$-such that its projective orbit $\left\{\lambda T^{n} x: \lambda \in \mathbb{K}, n \geq 1\right\}$ is dense in $X$. It is easy to extend this notion from the discrete to the continuous case: we say that a strongly continuous semigroup of operators $(T(t))_{t \geq 0}$ on $X$ is supercyclic whenever there is some vector $x \in X$ such that its projective orbit $\{\lambda T(t) x: \lambda \in \mathbb{K}, t \geq 0\}$ is dense in $X$. The following result shows that the earlier negative result also holds for semigroups.

Theorem 5.1. There is no supercyclic strongly continuous semigroup of operators on the space $\varphi$.

Proof. Suppose that $(T(t))_{t \geq 0}$ is a strongly continuous semigroup of operators on $\varphi$ having some supercyclic vector $x \in \varphi$. Then the projective orbit $\{\lambda T(t) x: \lambda \in$ $\mathbb{K}, t \geq 0\}$ is dense in $\varphi$. Since the set $\{\lambda T(0) x: \lambda \in \mathbb{K}\}=\{\lambda x: \lambda \in \mathbb{K}\}$ is nowhere dense, we have that $\operatorname{span}\{T(t) x: t>0\}$ is a dense subspace of $\varphi$. But every subspace of $\varphi$ is closed because $\varphi$ carries the finest locally convex topology. Consequently

$$
\varphi=\operatorname{span}\{T(t) x: t>0\} .
$$

In particular, there are finitely many scalars $a_{1}, \ldots, a_{m}$ with $a_{m} \neq 0$ and positive numbers $0<t_{1}<\cdots<t_{m}$ such that $x=\sum_{j=1}^{m} a_{j} T\left(t_{j}\right) x$. Hence, since $T(0) x=x$, there are scalars $b_{0}, \ldots, b_{m-1}$ such that

$$
T\left(t_{m}\right) x=\sum_{j=0}^{m-1} b_{j} T\left(t_{j}\right) x,
$$

where $t_{0}:=0$.

We now claim that

$$
\varphi=\operatorname{span}\left\{T(t) x: 0 \leq t \leq t_{m}\right\} .
$$

According to (1), it is enough to show that

$$
T(u) x \in \operatorname{span}\left\{T(t) x: 0 \leq t \leq t_{m}\right\}
$$


for all $u>0$. This is evident for $u \in\left(0, t_{m}\right]$. Let $0<h<\Delta$, where $\Delta=t_{m}-t_{m-1}>$ 0 . Then, by (2),

$$
T\left(t_{m}+h\right) x=T(h) T\left(t_{m}\right) x=\sum_{j=0}^{m-1} b_{j} T\left(t_{j}+h\right) x \in \operatorname{span}\left\{T(t) x: 0 \leq t \leq t_{m}\right\},
$$

hence (44) also holds for $u \in\left(0, t_{m}+\Delta\right]$.

Moreover, the above implies that

$$
\begin{aligned}
T(\Delta) \operatorname{span}\left\{T(t) x: 0 \leq t \leq t_{m}\right\} & \subset \operatorname{span}\left\{T(t+\Delta) x: 0 \leq t \leq t_{m}\right\} \\
& \subset \operatorname{span}\left\{T(t) x: 0 \leq t \leq t_{m}\right\} .
\end{aligned}
$$

Thus, $\operatorname{span}\left\{T(t) x: 0 \leq t \leq t_{m}\right\}$ is invariant under $T(\Delta)$. An induction procedure shows that (4) holds for all $u>0$, hence (3).

Now, since $(T(t))_{t \geq 0}$ is strongly continuous and the interval $\left[0, t_{m}\right]$ is compact, the set $\left\{T(t) x: 0 \leq t \leq t_{m}\right\}$ is compact, hence bounded in $\varphi$. Since $\varphi$ is the strict inductive limit of the spaces $E_{n}=\left\{\left(x_{1}, x_{2}, \ldots\right) \in \varphi: x_{j}=0\right.$ for all $\left.j>n\right\}, n \geq 1$, the set $\left\{T(t) x: 0 \leq t \leq t_{m}\right\}$ is in fact contained in some step $E_{n}$; see [32, p. 161]. In view of (3) this implies that $\varphi=E_{n}$, which is absurd. This contradiction proves the theorem.

Remark 5.2. In contrast, by Conejero [16] there do exist strongly continuous semigroups $(T(t))_{t \geq 0}$ on $\varphi$ that are topologically transitive, that is, for any pair of nonempty open subsets $U, V$ of $\varphi$ there exists $t \geq 0$ such that $T(t)(U) \cap V \neq \emptyset$. The analogous result for operators is due to Bonet, Frerick, Peris and Wengenroth 11.

Theorem 5.1] would follow directly from the corresponding result for operators if a suitable analogue of the Oxtoby-Ulam theorem mentioned in the Introduction were true. Thus we pose the following problem.

Problem 5.3. If $(T(t))_{t \geq 0}$ is a supercyclic (hypercyclic) strongly continuous semigroup on a complete LB-space, does it follow that for some $t>0$ the operator $T(t)$ is supercyclic (hypercyclic)?

We remark that, it is not even clear if the Oxtoby-Ulam theorem holds for supercyclic operators on F-spaces.

To end these notes we give an example to show that, in the larger class of (real) supercyclic semigroups, Problem 1.2 has a negative solution.

Example 5.4. There is a real supercyclic uniformly continuous semigroup $(T(t))_{t \geq 0}$ on Hilbert space such that some operator $T(t), t>0$, is not supercyclic. Such a semigroup is given by $T(t)=S(t) \oplus R(t), t \geq 0$, where $(S(t))_{t \geq 0}$ is a topologically mixing uniformly continuous semigroup on real Hilbert space $\bar{H}$ (see Remark 4.3), and $(R(t))_{t \geq 0}$ is the semigroup on $\mathbb{R}^{2}$ given by the matrices

$$
R(t)=\left(\begin{array}{cc}
\cos 2 \pi t & -\sin 2 \pi t \\
\sin 2 \pi t & \cos 2 \pi t
\end{array}\right) .
$$

We first show that the operator $T\left(t_{0}\right)$ is supercyclic whenever $t_{0}>0$ is irrational. This will then imply that $(T(t))_{t \geq 0}$ is a supercyclic semigroup. The supercyclicity of $T\left(t_{0}\right)$, in turn, follows once we have shown that, for any nonempty open subsets $U, V$ of $H$ and $\widetilde{U}, \widetilde{V}$ of $\mathbb{R}^{2}$, there are $\lambda \in \mathbb{R}$ and $n \in \mathbb{N}$ such that

$$
\lambda T\left(t_{0}\right)^{n}(U \oplus \widetilde{U}) \cap(V \oplus \widetilde{V}) \neq \emptyset
$$


(see [28, Theorem 1]). Indeed, we can choose $\rho, \sigma>0$ such that $\rho \widetilde{U} \cap S \neq \emptyset$ and $\sigma \widetilde{V} \cap S \neq \emptyset$, where $S$ denotes the unit sphere in $\mathbb{R}^{2}$. Now, since $(S(t))_{t \geq 0}$ is topologically mixing, there is some $N \in \mathbb{N}$ such that

$$
S\left(t_{0}\right)^{n}\left(\frac{\rho}{\sigma} U\right) \cap V \neq \emptyset \quad \text { for all } n \geq N .
$$

Moreover, since $t_{0}$ is irrational, there are $n_{1}, n_{2} \in \mathbb{N}$ with $n_{2} \geq n_{1}+N$ such that

$$
R\left(t_{0}\right)^{n_{1}}\left(\begin{array}{l}
1 \\
0
\end{array}\right) \in \rho \widetilde{U} \cap S \text { and } R\left(t_{0}\right)^{n_{2}}\left(\begin{array}{l}
1 \\
0
\end{array}\right) \in \sigma \widetilde{V} \cap S,
$$

hence

$$
\frac{1}{\rho} R\left(t_{0}\right)^{n_{1}}\left(\begin{array}{l}
1 \\
0
\end{array}\right) \in \widetilde{U} \quad \text { and } \quad \frac{\rho}{\sigma} R\left(t_{0}\right)^{n_{2}-n_{1}}\left(\frac{1}{\rho} R\left(t_{0}\right)^{n_{1}}\left(\begin{array}{l}
1 \\
0
\end{array}\right)\right) \in \widetilde{V} .
$$

This implies that

$$
\frac{\rho}{\sigma} R\left(t_{0}\right)^{n_{2}-n_{1}}(\widetilde{U}) \cap \widetilde{V} \neq \emptyset \text {. }
$$

By (6) we also have that

$$
\frac{\rho}{\sigma} S\left(t_{0}\right)^{n_{2}-n_{1}}(U) \cap V \neq \emptyset,
$$

so that (15) is satisfied with $\lambda=\frac{\rho}{\sigma}$ and $n=n_{2}-n_{1}$.

On the other hand, the operator $T(1)$ is clearly not supercyclic because $R(1)=I$.

\section{ACKNOWLEDGEMENT}

We are grateful to Antonio Bonilla for bringing to our attention the interesting paper 2. In addition we wish to thank Antonio Bonilla and Alfredo Peris for helpful discussions.

\section{REFERENCES}

[1] S. I. Ansari, Existence of hypercyclic operators on topological vectors spaces, J. Funct. Anal. 148 (1997), 384-390. MR1469346 (98h:47028a)

[2] T. Bermúdez, A. Bonilla, J. A. Conejero and A. Peris, Hypercyclic, topologically mixing and chaotic semigroups on Banach spaces, Studia Math. 170 (2005), 57-75. MR2142183 (2005m:47013)

[3] T. Bermúdez, A. Bonilla and A. Martinón, On the existence of chaotic and hypercyclic semigroups on Banach spaces, Proc. Amer. Math. Soc. 131 (2003), 2435-2441. MR.1974641 (2004b:47006)

[4] T. Bermúdez, A. Bonilla and A. Peris, On hypercyclicity and supercyclicity criteria, Bull. Austral. Math. Soc. 70 (2004), 45-54. MR2079359 (2005d:47014)

[5] L. Bernal-González, On hypercyclic operators on Banach spaces, Proc. Amer. Math. Soc. 127 (1999), 1003-1010. MR1476119 (99f:47010)

[6] L. Bernal-González and A. Bonilla, Exponential type of hypercyclic entire functions, Arch. Math. (Basel) 78 (2002), 283-290. MR.1895500(2002m:30033)

[7] L. Bernal-González and K.-G. Grosse-Erdmann, The Hypercyclicity Criterion for sequences of operators, Studia Math. 157 (2003), 17-32. MR.1980114 (2003m:47013)

[8] J. Bès and K. Chan, Denseness of hypercyclic operators on a Fréchet space, Houston J. Math. 29 (2003), 195-206. MR.1952504 (2003j:47006)

[9] J. Bès and K. Chan, Approximation by chaotic operators and by conjugate classes, J. Math. Anal. Appl. 284 (2003), 206-212. MR1996128 (2004j:47014)

[10] J. Bès and A. Peris, Hereditarily hypercyclic operators, J. Funct. Anal. 167 (1999), 94-112. MR.1710637 (2000f:47012)

[11] J. Bonet, L. Frerick, A. Peris and J. Wengenroth, Transitive and hypercyclic operators on locally convex spaces, Bull. London Math. Soc. 37 (2005), 254-264. MR2119025 (2005k:47021)

[12] J. Bonet, F. Martínez-Giménez and A. Peris, A Banach space which admits no chaotic operator, Bull. London Math. Soc. 33 (2001), 196-198. MR1815423 (2001m:47015)

[13] J. Bonet, F. Martínez-Giménez and A. Peris, Linear chaos on Fréchet spaces, Internat. J. Bifur. Chaos Appl. Sci. Engrg. 13 (2003), 1649-1655. MR2015614 (2004i:47016) 
[14] J. Bonet and A. Peris, Hypercyclic operators on non-normable Fréchet spaces, J. Funct. Anal. 159 (1998), 587-595. MR 1658096 (99k:47044)

[15] K. C. Chan and J. H. Shapiro, The cyclic behavior of translation operators on Hilbert spaces of entire functions, Indiana Univ. Math. J. 40 (1991), 1421-1449. MR1142722 (92m:47060)

[16] J. A. Conejero Casares, "Operadores y semigrupos de operadores en espacios de Fréchet y espacios localmente convexos", Ph.D. thesis, Universitat Politècnica de València, Valencia, 2004 .

[17] J. A. Conejero and A. Peris, Linear transitivity criteria, Topology Appl. 153 (2005), 767773. MR2203889

[18] G. Costakis and M. Sambarino, Topologically mixing hypercyclic operators, Proc. Amer. Math. Soc. 132 (2004), 385-389. MR2022360 (2004i:47017)

[19] W. Desch, W. Schappacher and G. F. Webb, Hypercyclic and chaotic semigroups of linear operators, Ergodic Theory Dynam. Systems 17 (1997), 793-819. MR1468101 (98j:47083)

[20] L. Dunford and J. T. Schwartz, "Linear Operators", Part I, Interscience, New York, 1958. MR 0117523(22:8302)

[21] S. El Mourchid, On a hypercyclicity criterion for strongly continuous semigroups, Discrete Contin. Dyn. Syst. 13 (2005), 271-275. MR2152389 (2006d:47017)

[22] K.-J. Engel and R. Nagel, "One-parameter semigroups for linear evolution equations", Springer, New York, 2000. MR1721989 (2000i:47075)

[23] R. M. Gethner and J. H. Shapiro, Universal vectors for operators on spaces of holomorphic functions, Proc. Amer. Math. Soc. 100 (1987), 281-288. MR0884467 (88g:47060)

[24] G. Godefroy and J. H. Shapiro, Operators with dense, invariant, cyclic vector manifolds, J. Funct. Anal. 98 (1991), 229-269. MR1111569 (92d:47029)

[25] S. Grivaux, Construction of operators with prescribed behaviour, Arch. Math. (Basel) 81 (2003), 291-299. MR2013260 (2004g:47011)

[26] S. Grivaux, Hypercyclic operators, mixing operators, and the bounded steps problem, J. Operator Theory 54 (2005), 147-168. MR2168865

[27] K.-G. Grosse-Erdmann, On the universal functions of G. R. MacLane, Complex Variables Theory Appl. 15 (1990), 193-196. MR.1074061 (91i:30021)

[28] K.-G. Grosse-Erdmann, Universal families and hypercyclic operators, Bull. Amer. Math. Soc. 36 (1999), 345-381. MR1685272 (2000c:47001)

[29] K.-G. Grosse-Erdmann, Hypercyclic and chaotic weighted shifts, Studia Math. 139 (2000), 47-68. MR1763044 (2001f:47051)

[30] K.-G. Grosse-Erdmann, Recent developments in hypercyclicity, RACSAM Rev. R. Acad. Cienc. Exactas Fís. Nat. Ser. A Mat. 97 (2003), 273-286. MR2068180 (2005c:47010)

[31] D. W. Hadwin, E. A. Nordgren, H. Radjavi and P. Rosenthal, Most similarity orbits are strongly dense, Proc. Amer. Math. Soc. 76 (1979), 250-252. MR0537082 (80i:47029)

[32] J. Horváth, "Topological vector spaces and distributions", vol. I, Addison-Wesley, Reading, 1966. MR 0205028 (34:4863)

[33] C. Kitai, "Invariant closed sets for linear operators", Ph.D. thesis, University of Toronto, Toronto, 1982.

[34] F. León-Saavedra, Notes about the hypercyclicity criterion, Math. Slovaca 53 (2003), 313319. MR2025025 (2004k:47010)

[35] F. León-Saavedra and A. Montes-Rodríguez, Linear structure of hypercyclic vectors, J. Funct. Anal. 148 (1997), 524-545. MR1469352 (98h:47028b)

[36] F. Martínez-Giménez and A. Peris, Chaos for backward shift operators, Internat. J. Bifur. Chaos Appl. Sci. Engrg. 12 (2002), 1703-1715. MR.1927407(2003h:47056)

[37] V. Müller, "Spectral theory of linear operators and spectral systems in Banach algebras", Birkhäuser Verlag, Basel, 2003. MR 1975356 (2004c:47008)

[38] G. A. Muñoz, Y. Sarantopoulos and A. Tonge, Complexifications of real Banach spaces, polynomials and multilinear maps, Studia Math. 134 (1999), 1-33. MR.1688213 (2000g:46009)

[39] R. I. Ovsepian and A. Pełczyński, On the existence of a fundamental total and bounded biorthogonal sequence in every separable Banach space, and related constructions of uniformly bounded orthonormal systems in $L^{2}$, Studia Math. 54 (1975), 149-159. MR0394137 $(52: 14942)$

[40] J. C. Oxtoby and S. M. Ulam, Measure-preserving homeomorphisms and metrical transitivity, Ann. of Math. (2) 42 (1941), 874-920. MR0005803 (3:211b) 
[41] A. Peris, Hypercyclicity criteria and the Mittag-Leffler theorem, Bull. Soc. Roy. Sci. Liège 70 (2001), 365-371. MR1904062 (2003c:47012)

[42] A. Peris and L. Saldivia, Syndetically hypercyclic operators, Integral Equations Operator Theory 51 (2005), 275-281. MR2120081 (2005h:47017)

[43] S. Rolewicz, On orbits of elements, Studia Math. 32 (1969), 17-22. MR0241956 (39:3292)

[44] W. Rudin, "Functional Analysis", 2nd ed., McGraw-Hill, New York, 1991. MR.1157815 (92k:46001)

Departamento de Análisis Matemático, Facultad de Matemáticas, Apdo. 1160, Avda. Reina Mercedes, 41080 Sevilla, Spain

E-mail address: lbernal@us.es

Fachbereich Mathematik, Fernuniversität Hagen, 58084 Hagen, Germany

E-mail address: kg.grosse-erdmann@fernuni-hagen.de 